\title{
Enstrophy transfers in helical turbulence
}

\author{
Shubhadeep Sadhukhan, ${ }^{1, *}$ Roshan Samuel, ${ }^{2, \dagger}$ Franck Plunian $\odot,{ }^{3, \ddagger}$ Rodion Stepanov,,${ }^{4, \S}$ \\ Ravi Samtaney, ${ }^{5, \|}$ and Mahendra Kumar Verma ${ }^{1, \mathbb{\Phi}}$ \\ ${ }^{1}$ Department of Physics, Indian Institute of Technology, Kanpur, Uttar Pradesh 208016, India \\ ${ }^{2}$ Department of Mechanical Engineering, Indian Institute of Technology, Kanpur 208016, India \\ ${ }^{3}$ Université Grenoble Alpes, Université Savoie Mont Blanc, CNRS, IRD, IFSTTAR, \\ ISTerre, 38000 Grenoble, France \\ ${ }^{4}$ Institute of Continuous Media Mechanics UB RAS, Perm, Russian Federation \\ ${ }^{5}$ Mechanical Engineering, Division of Physical Science and Engineering, \\ King Abdullah University of Science and Technology, Thuwal 23955-6900, Kingdom of Saudi Arabia
}

(Received 10 April 2019; published 28 August 2019)

\begin{abstract}
In this paper we study the enstrophy transfers in helical turbulence using direct numerical simulation. We observe that the helicity injection does not have significant effects on the inertial-range energy and helicity spectra $\left(\sim k^{-5 / 3}\right)$ and fluxes (constants). We also calculate the separate contributions to enstrophy transfers via velocity-to-vorticity and vorticity-tovorticity channels. There are four different enstrophy fluxes associated with the former channel or vorticity stretching, and one flux associated with the latter channel or vorticity advection. In the inertial range, the fluxes due to vorticity stretching are larger than that due to advection. These transfers too are insensitive to helicity injection.
\end{abstract}

DOI: 10.1103/PhysRevFluids.4.084607

\section{INTRODUCTION}

Turbulence is a classic and still open problem in fluid dynamics. The energetics of homogeneous and isotropic turbulence were explained through the celebrated works of Kolmogorov [1,2]. Kolmogorov argued that under statistically steady state, the rate of energy supplied by an external force is equal to the rate at which energy cascades from large to smaller scales, which is equal to the rate of energy dissipation. Obukhov [3] and Corrsin [4] generalized Kolmogorov's theory to isotropic turbulence with statistically homogeneous fluctuations of the temperature field embedded in a turbulent flow. The theories of Kolmogorov, Obukhov, and Corrsin, collectively referred to as Kolmogorov-Obukhov theory, predict that the kinetic energy spectrum $E(k)=u_{k}^{2} / k \sim k^{-5 / 3}$, and the spectrum of temperature fluctuations $E_{\zeta}(k)=\zeta_{k}^{2} / k \sim k^{-5 / 3}$, where $k=2 \pi / l$ is the wave number with $l$ as the length scale, and $u_{k}$ and $\zeta_{k}$ are respectively the velocity and temperature fluctuations at wave number $k$. Researchers have also studied the spectra of density and other passive scalars in the same framework. Refer to Lesieur [5] and Verma [6] for detailed references. It is important to note that the effect of helicity is absent in the above-described works.

\footnotetext{
*deep@iitk.ac.in

†roshanj@iitk.ac.in

†ranck.plunian@univ-grenoble-alpes.fr

§rodion@icmm.ru

"ravi.samtaney@kaust.edu.sa

Imkv@iitk.ac.in
} 
Energy and helicity are two inviscid conserved quantities in three-dimensional (3D) turbulent flows. The effect of helicity in fully developed 3D turbulence has been extensively studied [7-25]. André and Lesieur [9] studied the effect of helicity on the evolution of isotropic turbulence at high Reynolds numbers using a variant of Markovian eddy-damped quasinormal (EDQNM) theory. Polifke and Shtilman [10] showed that the energy decay is slowed down by large initial helicity. Waleffe [11] introduced helical decomposition of the velocity field and discussed triadic interactions in helical flows. Yokoi and Yoshizawa [12] reported the effects of helicity in 3D incompressible inhomogeneous turbulence with the help of a two-scale direct-interaction approximation (DIA). Zhou and Vahala [13] showed that helicity does not alter renormalized viscosity. Ditlevsen and Giuliani [14], Chen et al. [15], and Lessinnes et al. [16] studied helicity fluxes for different types of helical modes. Chen et al. [17] studied intermittency in helical turbulence. Teitelbaum and Mininni [18] explored the effect of helicity in rotating turbulent flows, while Pouquet et al. [19] reported evidence for three-dimensionalization recovered at small scales. Stepanov et al. [20] explored the helical bottleneck effect in 3D isotropic and homogeneous turbulence using a shell model. Maximum helicity states have been studied by Biferale et al. [21], Kessar et al. [22], and Sahoo and Biferale [23]. Mode-to-mode and shell-to-shell helicity transfers have been studied by Avinash et al. [24] and Teimurazov et al. [25]. Helicity may also play a crucial role in magnetohydrodynamics [26-28] and dynamo action [29-32].

In this paper we derive formulas for mode-to-mode enstrophy transfers and their associated fluxes. These quantities arise due to the nonlinear interactions. In Sec. II we derive the expressions of mode-to-mode enstrophy transfers and the corresponding fluxes. In Sec. III, after introducing the helical forcing that we use, the enstrophy fluxes are calculated from direct simulations of 3D turbulence using the pseudospectral code TARANG [33,34]. We conclude in Sec. IV.

\section{ENSTROPHY TRANSFERS AND FLUXES}

The motion of an incompressible fluid is described by the Navier-Stokes equations

$$
\begin{gathered}
\frac{\partial \mathbf{u}}{\partial t}=-(\mathbf{u} \cdot \nabla) \mathbf{u}-\nabla p+v \nabla^{2} \mathbf{u}+\mathbf{F}, \\
\nabla \cdot \mathbf{u}=0,
\end{gathered}
$$

where $\mathbf{u}$ and $p$ are the velocity and pressure fields, $v$ the kinematic viscosity, and $\mathbf{F}$ an external force.

The vorticity $\boldsymbol{\omega}=\nabla \times \mathbf{u}$ obeys the following equation:

$$
\frac{\partial \boldsymbol{\omega}}{\partial t}=(\boldsymbol{\omega} \cdot \nabla) \mathbf{u}-(\mathbf{u} \cdot \nabla) \boldsymbol{\omega}+v \nabla^{2} \boldsymbol{\omega}+\nabla \times \mathbf{F},
$$

where the first two terms in the right-hand side correspond to vorticity stretching and vorticity advection respectively. In Fourier space the vorticity, which is defined as $\boldsymbol{\omega}(\mathbf{k})=i \mathbf{k} \times \boldsymbol{u}(\mathbf{k})$, satisfies

$$
\frac{d}{d t} \boldsymbol{\omega}(\mathbf{k})=i \sum_{\mathbf{p}}(\mathbf{k} \cdot \boldsymbol{\omega}(\mathbf{q})) \mathbf{u}(\mathbf{p})-(\mathbf{k} \cdot \mathbf{u}(\mathbf{q})) \boldsymbol{\omega}(\mathbf{p})-v k^{2} \boldsymbol{\omega}(\mathbf{k})+i \mathbf{k} \times \mathbf{F}(\mathbf{k}),
$$

where $\mathbf{q}=\mathbf{k}-\mathbf{p}$ and $k=|\mathbf{k}|$.

\section{A. Enstrophy transfers}

Enstrophy, which is defined as $W(\mathbf{k})=|\boldsymbol{\omega}(\mathbf{k})|^{2} / 2$, satisfies

$$
\begin{aligned}
\frac{d}{d t} W(\mathbf{k})= & \sum_{\mathbf{p}}\left[\operatorname{Im}\left\{[\mathbf{k} \cdot \mathbf{u}(\mathbf{q})]\left[\omega(\mathbf{p}) \cdot \boldsymbol{\omega}^{*}(\mathbf{k})\right]\right\}-\operatorname{Im}\left\{[\mathbf{k} \cdot \boldsymbol{\omega}(\mathbf{q})]\left[\mathbf{u}(\mathbf{p}) \cdot \omega^{*}(\mathbf{k})\right]\right\}\right] \\
& -2 v k^{2} W(\mathbf{k})+k^{2} \operatorname{Re}\left\{\mathbf{u}^{*}(\mathbf{k}) \cdot \mathbf{F}(\mathbf{k})\right\},
\end{aligned}
$$


where $\mathbf{q}=\mathbf{k}-\mathbf{p}$. Setting $\mathbf{k}^{\prime}=-\mathbf{k}$, the above equation can be rewritten in the form

$$
\frac{d}{d t} W\left(\mathbf{k}^{\prime}\right)=\sum_{\mathbf{p}}\left[S^{\omega \omega}\left(\mathbf{k}^{\prime} \mid \mathbf{p}, \mathbf{q}\right)+S^{\omega u}\left(\mathbf{k}^{\prime} \mid \mathbf{p}, \mathbf{q}\right)\right]-2 \nu k^{\prime 2} W\left(\mathbf{k}^{\prime}\right)+k^{\prime 2} \operatorname{Re}\left\{\mathbf{u}^{*}\left(\mathbf{k}^{\prime}\right) \cdot \mathbf{F}\left(\mathbf{k}^{\prime}\right)\right\}
$$

where $S^{\omega \omega}\left(\mathbf{k}^{\prime} \mid \mathbf{p}, \mathbf{q}\right)$ and $S^{\omega u}\left(\mathbf{k}^{\prime} \mid \mathbf{p}, \mathbf{q}\right)$ are the combined transfers of enstrophy from modes $\mathbf{p}$ and $\mathbf{q}$ to $\mathbf{k}^{\prime}$, defined as

$$
\begin{gathered}
S^{\omega \omega}\left(\mathbf{k}^{\prime} \mid \mathbf{p}, \mathbf{q}\right)=-\operatorname{Im}\left\{\left[\mathbf{k}^{\prime} \cdot \mathbf{u}(\mathbf{q})\right]\left[\omega(\mathbf{p}) \cdot \omega\left(\mathbf{k}^{\prime}\right)\right]\right\}-\operatorname{Im}\left\{\left[\mathbf{k}^{\prime} \cdot \mathbf{u}(\mathbf{p})\right]\left[\omega(\mathbf{q}) \cdot \omega\left(\mathbf{k}^{\prime}\right)\right]\right\} \\
S^{\omega u}\left(\mathbf{k}^{\prime} \mid \mathbf{p}, \mathbf{q}\right)=+\operatorname{Im}\left\{\left[\mathbf{k}^{\prime} \cdot \omega(\mathbf{q})\right]\left[\mathbf{u}(\mathbf{p}) \cdot \omega\left(\mathbf{k}^{\prime}\right)\right]\right\}+\operatorname{Im}\left\{\left[\mathbf{k}^{\prime} \cdot \omega(\mathbf{p})\right]\left[\mathbf{u}(\mathbf{q}) \cdot \boldsymbol{\omega}\left(\mathbf{k}^{\prime}\right)\right]\right\}
\end{gathered}
$$

with $\mathbf{k}^{\prime}+\mathbf{p}+\mathbf{q}=0$. Using the incompressibility condition, we can show that

$$
S^{\omega \omega}\left(\mathbf{k}^{\prime} \mid \mathbf{p}, \mathbf{q}\right)+S^{\omega \omega}\left(\mathbf{p} \mid \mathbf{q}, \mathbf{k}^{\prime}\right)+S^{\omega \omega}\left(\mathbf{q} \mid \mathbf{p}, \mathbf{k}^{\prime}\right)=0,
$$

where $S^{\omega \omega}$ represents the advection of vortices, thus involving enstrophy exchange among the vorticity modes $\omega\left(\mathbf{k}^{\prime}\right), \boldsymbol{\omega}(\mathbf{p})$, and $\boldsymbol{\omega}(\mathbf{q})$. On the other hand we have

$$
S^{\omega u}\left(\mathbf{k}^{\prime} \mid \mathbf{p}, \mathbf{q}\right)+S^{\omega u}\left(\mathbf{p} \mid \mathbf{q}, \mathbf{k}^{\prime}\right)+S^{\omega u}\left(\mathbf{q} \mid \mathbf{k}^{\prime}, \mathbf{p}\right) \neq 0,
$$

where $S^{\omega u}$ represents the stretching of vortices, thus either increasing or decreasing the enstrophy $E_{\omega}$. Incidentally (10) implies that enstrophy is not a conserved quantity in 3D hydrodynamics.

Now we can split the above transfers into individual contributions from modes $\mathbf{p}$ and $\mathbf{q}$ :

$$
\begin{aligned}
& S^{\omega \omega}\left(\mathbf{k}^{\prime} \mid \mathbf{p}, \mathbf{q}\right)=S^{\omega \omega}\left(\mathbf{k}^{\prime}|\mathbf{p}| \mathbf{q}\right)+S^{\omega \omega}\left(\mathbf{k}^{\prime}|\mathbf{q}| \mathbf{p}\right), \\
& S^{\omega u}\left(\mathbf{k}^{\prime} \mid \mathbf{p}, \mathbf{q}\right)=S^{\omega u}\left(\mathbf{k}^{\prime}|\mathbf{p}| \mathbf{q}\right)+S^{\omega u}\left(\mathbf{k}^{\prime}|\mathbf{q}| \mathbf{p}\right),
\end{aligned}
$$

with

$$
\begin{aligned}
& S^{\omega \omega}\left(\mathbf{k}^{\prime}|\mathbf{p}| \mathbf{q}\right)=-\operatorname{Im}\left\{\left[\mathbf{k}^{\prime} \cdot \mathbf{u}(\mathbf{q})\right]\left[\omega(\mathbf{p}) \cdot \omega\left(\mathbf{k}^{\prime}\right)\right]\right\}, \\
& S^{\omega u}\left(\mathbf{k}^{\prime}|\mathbf{p}| \mathbf{q}\right)=+\operatorname{Im}\left\{\left[\mathbf{k}^{\prime} \cdot \boldsymbol{\omega}(\mathbf{q})\right]\left[\mathbf{u}(\mathbf{p}) \cdot \omega\left(\mathbf{k}^{\prime}\right)\right]\right\} .
\end{aligned}
$$

Here $S^{\omega \omega}\left(\mathbf{k}^{\prime}|\mathbf{p}| \mathbf{q}\right)$ and $S^{\omega u}\left(\mathbf{k}^{\prime}|\mathbf{p}| \mathbf{q}\right)$ denote two kinds of mode-to-mode enstrophy transfer, both being from $\mathbf{p}$ to $\mathbf{k}^{\prime}$ with $\mathbf{q}$ acting as a mediator. They arise due to advection and stretching respectively. Here we note that

$$
S^{\omega \omega}\left(\mathbf{k}^{\prime}|\mathbf{p}| \mathbf{q}\right)=-S^{\omega \omega}\left(\mathbf{p}\left|\mathbf{k}^{\prime}\right| \mathbf{q}\right)
$$

due to the incompressibility condition, $\mathbf{k} \cdot \mathbf{u}(\mathbf{k})=0$; this result is consistent with (9). Substitution of (13) and (14) into (5) yields

$$
\frac{d}{d t} W\left(\mathbf{k}^{\prime}\right)=\sum_{\mathbf{p}} S^{\omega \omega}\left(\mathbf{k}^{\prime}|\mathbf{p}| \mathbf{q}\right)+\sum_{\mathbf{p}} S^{\omega u}\left(\mathbf{k}^{\prime}|\mathbf{p}| \mathbf{q}\right)-2 \nu k^{\prime 2} W\left(\mathbf{k}^{\prime}\right)+k^{\prime 2} \operatorname{Re}\left\{\mathbf{u}^{*}\left(\mathbf{k}^{\prime}\right) \cdot \mathbf{F}\left(\mathbf{k}^{\prime}\right)\right\},
$$

and $\mathbf{q}=-\left(\mathbf{k}^{\prime}+\mathbf{p}\right)$.

\section{B. Enstrophy fluxes}

Summing the previously discussed mode-to-mode enstrophy transfers over $\mathbf{p}$ and $\mathbf{k}^{\prime}$ and depending on whether $\mathbf{p}$ and $\mathbf{k}^{\prime}$ belong to the sphere of radius $k_{0}$ or not, we can define the five 


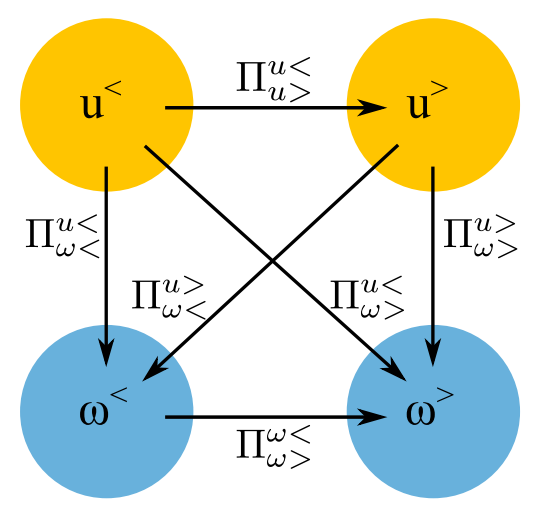

FIG. 1. Illustration of the five enstrophy fluxes in addition to the kinetic energy flux $\Pi_{u<}^{u>} \equiv \Pi_{E}$ which is defined in Eq. (39).

following enstrophy fluxes:

$$
\begin{aligned}
& \Pi_{\omega>}^{\omega<}\left(k_{0}\right)=\sum_{|\mathbf{p}| \leqslant k_{0}} \sum_{\left|\mathbf{k}^{\prime}\right|>k_{0}} S^{\omega \omega}\left(\mathbf{k}^{\prime}|\mathbf{p}| \mathbf{q}\right), \\
& \Pi_{\omega>}^{u<}\left(k_{0}\right)=\sum_{|\mathbf{p}| \leqslant k_{0}} \sum_{\left|\mathbf{k}^{\prime}\right|>k_{0}} S^{\omega u}\left(\mathbf{k}^{\prime}|\mathbf{p}| \mathbf{q}\right), \\
& \Pi_{\omega<}^{u<}\left(k_{0}\right)=\sum_{|\mathbf{p}| \leqslant k_{0}} \sum_{\left|\mathbf{k}^{\prime}\right| \leqslant k_{0}} S^{\omega u}\left(\mathbf{k}^{\prime}|\mathbf{p}| \mathbf{q}\right), \\
& \Pi_{\omega>}^{u>}\left(k_{0}\right)=\sum_{|\mathbf{p}|>k_{0}} \sum_{\left|\mathbf{k}^{\prime}\right|>k_{0}} S^{\omega u}\left(\mathbf{k}^{\prime}|\mathbf{p}| \mathbf{q}\right), \\
& \Pi_{\omega<}^{u>}\left(k_{0}\right)=\sum_{|\mathbf{p}|>k_{0}} \sum_{\left|\mathbf{k}^{\prime}\right| \leqslant k_{0}} S^{\omega u}\left(\mathbf{k}^{\prime}|\mathbf{p}| \mathbf{q}\right),
\end{aligned}
$$

where the superscript and subscript of $\Pi$ represent respectively the giver and the receiver modes, and the symbols $<$ and $>$ denote respectively the inside and outside of the sphere of radius $k_{0}$. Accordingly, $\Pi_{y>}^{x<}\left(k_{0}\right)$ denotes the flux of enstrophy from all $x$ modes inside the sphere of radius $k_{0}$ to all $y$ modes outside the sphere. An illustration of these fluxes is given in Fig. 1.

Customarily a turbulent flux is associated with some conserved quantity [5]. For example, in two-dimensional hydrodynamic turbulence, the energy and enstrophy fluxes are connected to the corresponding conservation laws. The concept of turbulent flux could be generalized to a more complex scenario when a certain quantity is transferred from one field to another. Here, these fluxes are cross transfers among the two fields. Though enstrophy is not conserved, we define enstrophy fluxes of Eqs. (18)-(21) as enstrophy transfers from large (small) scale velocity field to large (small) vorticity field. The enstrophy flux $\Pi_{\omega>}^{\omega<}\left(k_{0}\right)$ is related to the conservation law related to Eq. (9). Similar issues arise in magnetohydrodynamic turbulence where fluxes for kinetic and magnetic energies are defined even though kinetic and magnetic energies are not conserved individually. We define kinetic-to-magnetic energy fluxes as the energy transfers from large (small) scale velocity field to from large (small) scale magnetic field; such fluxes are crucial for dynamo action $[26,27,35]$.

Summing (16) over all modes outside the sphere of radius $k_{0}$, we obtain

$$
\sum_{\left|\mathbf{k}^{\prime}\right|>k_{0}}\left(\frac{d}{d t}+2 v k^{\prime 2}\right) W\left(\mathbf{k}^{\prime}\right)-k^{\prime 2} \operatorname{Re}\left\{\mathbf{u}^{*}\left(\mathbf{k}^{\prime}\right) \cdot \mathbf{F}\left(\mathbf{k}^{\prime}\right)\right\}=\left(\Pi_{\omega>}^{\omega<}+\Pi_{\omega>}^{u<}+\Pi_{\omega>}^{u>}\right)\left(k_{0}\right) .
$$


Performing a sum over all modes inside the sphere of radius $k_{0}$ yields

$$
\sum_{\left|\mathbf{k}^{\prime}\right| \leqslant k_{0}}\left(\frac{d}{d t}+2 v k^{\prime 2}\right) W\left(\mathbf{k}^{\prime}\right)-k^{\prime 2} \operatorname{Re}\left\{\mathbf{u}^{*}\left(\mathbf{k}^{\prime}\right) \cdot \mathbf{F}\left(\mathbf{k}^{\prime}\right)\right\}=\left(\Pi_{\omega<}^{\omega>}+\Pi_{\omega<}^{u<}+\Pi_{\omega<}^{u>}\right)\left(k_{0}\right) .
$$

Adding (22) and (23) and noticing that (15) implies $\Pi_{\omega>}^{\omega<}+\Pi_{\omega<}^{\omega>}=0$, we obtain

$$
\sum_{\mathbf{k}^{\prime}}\left(\frac{d}{d t}+2 \nu k^{\prime 2}\right) W\left(\mathbf{k}^{\prime}\right)-k^{\prime 2} \operatorname{Re}\left\{\mathbf{u}^{*}\left(\mathbf{k}^{\prime}\right) \cdot \mathbf{F}\left(\mathbf{k}^{\prime}\right)\right\}=\left(\Pi_{\omega>}^{u<}+\Pi_{\omega>}^{u>}+\Pi_{\omega<}^{u<}+\Pi_{\omega<}^{u>}\right)\left(k_{0}\right) .
$$

As the left-hand side of (24) does not depend on $k_{0}$, it implies

$$
\frac{d}{d k_{0}}\left(\Pi_{\omega>}^{u<}+\Pi_{\omega>}^{u>}+\Pi_{\omega<}^{u<}+\Pi_{\omega<}^{u>}\right)=0,
$$

which is valid at all times. That is, the sum

$$
\Pi_{\omega>}^{u<}\left(k_{0}\right)+\Pi_{\omega>}^{u>}\left(k_{0}\right)+\Pi_{\omega<}^{u<}\left(k_{0}\right)+\Pi_{\omega<}^{u>}\left(k_{0}\right)=\mathrm{const}
$$

is independent of $k_{0}$.

Under a steady state, we obtain

$$
\sum_{\mathbf{k}^{\prime}} 2 \nu k^{\prime 2} W\left(\mathbf{k}^{\prime}\right)-\sum_{k_{0}^{F}<\left|\mathbf{k}^{\prime}\right| \leqslant k_{1}^{F}} k^{\prime 2} \operatorname{Re}\left\{\mathbf{u}^{*}\left(\mathbf{k}^{\prime}\right) \cdot \mathbf{F}\left(\mathbf{k}^{\prime}\right)\right\}=\left(\Pi_{\omega>}^{u<}+\Pi_{\omega>}^{u>}+\Pi_{\omega<}^{u<}+\Pi_{\omega<}^{u>}\right)\left(k_{0}\right),
$$

where the forcing is employed to the wave number band $\left(k_{0}^{F}, k_{1}^{F}\right]$. A physical interpretation of the above equation is that the enstrophy injected by the external force is transferred to the enstrophy fluxes and the enstrophy dissipation.

\section{SIMULATION METHOD AND RESULTS}

The Navier-Stokes equations (1) are solved numerically using the fully dealiased, parallel pseudospectral code TARANG [33,34] with fourth-order Runge-Kutta time stepping. For dealiasing purpose, the $3 / 2$ rule has been chosen $[36,37]$. The viscosity is set to $v=10^{-3}$ and the simulations are performed with a resolution of $512^{3}$.

\section{A. Helical forcing}

In Fourier space the velocity field satisfies

$$
\frac{d}{d t} \mathbf{u}(\mathbf{k})=-i \sum_{\mathbf{p}}[\mathbf{k} \cdot \mathbf{u}(\mathbf{q})] \mathbf{u}(\mathbf{p})-v k^{2} \mathbf{u}(\mathbf{k})+\mathbf{F}(\mathbf{k}),
$$

where $\mathbf{q}=\mathbf{k}-\mathbf{p}$. Then the energy and helicity, which are defined as

$$
E(\mathbf{k})=\frac{|\mathbf{u}(\mathbf{k})|^{2}}{2}, \quad H(\mathbf{k})=\frac{\mathbf{u}(\mathbf{k}) \cdot \omega(\mathbf{k})^{*}}{2},
$$

satisfy the following equations:

$$
\begin{aligned}
\frac{d}{d t} E\left(\mathbf{k}^{\prime}\right) & =-\sum_{\mathbf{p}} \operatorname{Im}\left\{\left[\mathbf{k}^{\prime} \cdot \mathbf{u}(\mathbf{q})\left[\mathbf{u}(\mathbf{p}) \cdot \mathbf{u}\left(\mathbf{k}^{\prime}\right)\right]\right\}-2 v k^{2} E\left(\mathbf{k}^{\prime}\right)+\operatorname{Re}\left\{\mathbf{u}^{*}\left(\mathbf{k}^{\prime}\right) \cdot \mathbf{F}\left(\mathbf{k}^{\prime}\right)\right\},\right. \\
\frac{d}{d t} H\left(\mathbf{k}^{\prime}\right) & =\sum_{\mathbf{p}} \operatorname{Re}\left\{\mathbf{u}(\mathbf{q}) \cdot\left[\omega(\mathbf{p}) \times \omega\left(\mathbf{k}^{\prime}\right)\right]\right\}-2 v k^{2} H\left(\mathbf{k}^{\prime}\right)+\operatorname{Re}\left\{\omega^{*}\left(\mathbf{k}^{\prime}\right) \cdot \mathbf{F}\left(\mathbf{k}^{\prime}\right)\right\}
\end{aligned}
$$

where $\mathbf{q}=-\left(\mathbf{k}^{\prime}+\mathbf{p}\right)$. 
Following Carati et al. [38,39] and Teimurazov et al. [25], the forcing is taken to be of the form

$$
\mathbf{F}(\mathbf{k})=\alpha \mathbf{u}(\mathbf{k})+\beta \boldsymbol{\omega}(\mathbf{k})
$$

such that

$$
\sum_{k_{0}^{F}<|\mathbf{k}| \leqslant k_{1}^{F}} \operatorname{Re}\left\{\mathbf{u}^{*}(\mathbf{k}) \cdot \mathbf{F}(\mathbf{k})\right\}=\epsilon_{E}
$$

and

$$
\sum_{k_{0}^{F}<|\mathbf{k}| \leqslant k_{1}^{F}} \operatorname{Re}\left\{\boldsymbol{\omega}^{*}(\mathbf{k}) \cdot \mathbf{F}(\mathbf{k})\right\}=\epsilon_{H} .
$$

Here, the forcing is applied in the wave number band $\left(k_{0}^{F}, k_{1}^{F}\right]$, and $\epsilon_{E}$ and $\epsilon_{H}$ are the prescribed energy and helicity injection rates. From the above three equations (32)-(34) we deduce that

$$
\alpha=\frac{1}{2} \frac{W_{F} \epsilon_{E}-H_{F} \epsilon_{H}}{E_{F} W_{F}-H_{F}^{2}}, \quad \beta=\frac{1}{2} \frac{E_{F} \epsilon_{H}-H_{F} \epsilon_{E}}{E_{F} W_{F}-H_{F}^{2}},
$$

where $E_{F}, H_{F}$, and $W_{F}$ are respectively the energy, kinetic helicity and enstrophy in the forcing band,

$$
\left(E_{F}, H_{F}, W_{F}\right)=\sum_{k_{0}^{F}<|\mathbf{k}| \leqslant k_{1}^{F}}(E(\mathbf{k}), H(\mathbf{k}), W(\mathbf{k})) .
$$

In our simulations, the forcing band corresponds to $k_{0}^{F}=2$ and $k_{1}^{F}=3$. The main advantage of using the forcing given by (32)-(35) is that the injection rates of energy and helicity can be set independently. The realizability condition $|H(\mathbf{k})| \leqslant k E(\mathbf{k})$ directly comes from the definitions of energy and helicity and is therefore always satisfied, whatever the values of $\epsilon_{E}$ and $\epsilon_{H}$. For example even in the case $\epsilon_{E} \ll \epsilon_{H}$, which was studied by Kessar et al. [22], the realizability condition is satisfied, reaching a state close to the maximal helical state $|H(\mathbf{k})|=k E(\mathbf{k})$.

In Fig. 2 the time evolution of (a) the total energy dissipation $2 v \sum_{\mathbf{k}} k^{2} E(\mathbf{k})$, (b) the total helicity dissipation $2 v \sum_{\mathbf{k}} k^{2} H(\mathbf{k})$, and (c) the total enstrophy dissipation $2 v \sum_{\mathbf{k}} k^{2} W(\mathbf{k})$ are plotted for the same energy injection rate $\epsilon_{E}=0.1$ and three helicity injections $\epsilon_{H}=0,0.1,0.2$. We observe that a statistical steady state is reached for $t \geqslant 10$. In this steady state we find that, on average, $2 v \sum_{\mathbf{k}} k^{2} E(\mathbf{k}) \approx \epsilon_{E}$ and $2 v \sum_{\mathbf{k}} k^{2} H(\mathbf{k}) \approx \epsilon_{H}$, which is consistent with the prescribed forcing.

\section{B. Energy and helicity spectra and fluxes}

From (30) and (31) and following steps analogous to (5)-(14), the following expression for modeto-mode energy and helicity transfers can be derived:

$$
\begin{gathered}
S^{E}\left(\mathbf{k}^{\prime}|\mathbf{p}| \mathbf{q}\right)=-\operatorname{Im}\left\{\left[\mathbf{k}^{\prime} \cdot \mathbf{u}(\mathbf{q})\right]\left[\mathbf{u}(\mathbf{p}) \cdot \mathbf{u}\left(\mathbf{k}^{\prime}\right)\right]\right\}, \\
S^{H}\left(\mathbf{k}^{\prime}|\mathbf{p}| \mathbf{q}\right)=+\operatorname{Re}\left\{\mathbf{u}(\mathbf{q}) \cdot\left[\boldsymbol{\omega}(\mathbf{p}) \times \boldsymbol{\omega}\left(\mathbf{k}^{\prime}\right)\right]\right\},
\end{gathered}
$$

leading to the flux definitions

$$
\Pi_{E, H}\left(k_{0}\right)=\sum_{\left|\mathbf{k}^{\prime}\right|>k_{0}} \sum_{|\mathbf{p}|<k_{0}} S^{E, H}\left(\mathbf{k}^{\prime}|\mathbf{p}| \mathbf{q}\right) .
$$

In Fig. 3, the energy and helicity spectra, normalized by respectively $\epsilon_{E}$ and $\epsilon_{H}$, show a spectral scaling close to $k^{-\frac{5}{3}}$ in the inertial range, for the three injection rates of helicity $\epsilon_{H}=0,0.1,0.2$. The normalized fluxes shown in the inset are approximately flat in the inertial range, and again insensitive to $\epsilon_{H}$. 


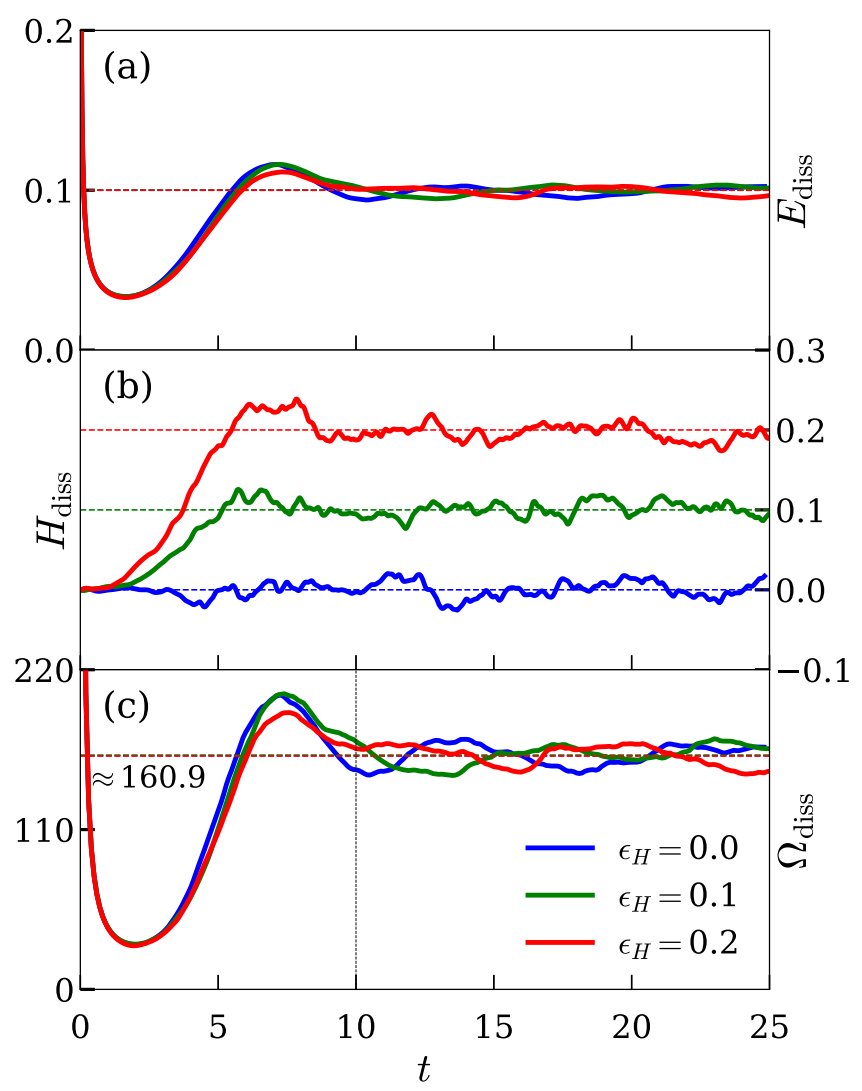

FIG. 2. Time evolution of (a) energy dissipation, (b) helicity dissipation, and (c) enstrophy dissipation for the same energy injection rate $\epsilon_{E}=0.1$ and three different helicity injections $\epsilon_{H}=0,0.1,0.2$. For $t \geqslant 10$ a steady state is reached with a mean enstrophy dissipation about 160.9 .

In Fig. 3 the black dashed curves correspond to the analytical formulas for spectra and fluxes derived by Pao [40] for the energy and that are here extended to helicity. They take the following forms:

$$
\begin{gathered}
\frac{E(k)}{\epsilon_{E}}=\frac{K_{E}}{\epsilon_{E}^{1 / 3}} k^{-5 / 3} \exp \left(-\frac{3}{2} K_{E}\left(k / k_{d}\right)^{4 / 3}\right), \\
\frac{H(k)}{\epsilon_{H}}=\frac{K_{H}}{\epsilon_{E}^{1 / 3}} k^{-5 / 3} \exp \left(-\frac{3}{2} K_{H}\left(k / k_{d}\right)^{4 / 3}\right), \\
\frac{\Pi_{E}(k)}{\epsilon_{E}}=\exp \left(-\frac{3}{2} K_{E}\left(k / k_{d}\right)^{4 / 3}\right), \\
\frac{\Pi_{H}(k)}{\epsilon_{H}}=\exp \left(-\frac{3}{2} K_{H}\left(k / k_{d}\right)^{4 / 3}\right),
\end{gathered}
$$

where $K_{E}$ is Kolmogorov's constant, $K_{H}$ is another nondimensional constants and $k_{d}=\left(\epsilon_{E} / v^{3}\right)^{1 / 4}$ is Kolmogorov's wave number. Note, however, that Pao's spectrum slightly overestimates the dissipation range spectrum [41], and we expect similar discrepancies for the kinetic helicity that may show up in high-resolution simulations. These issues may be related to intermittency and enhanced dissipation due to the bottleneck effect $[42,43]$. 


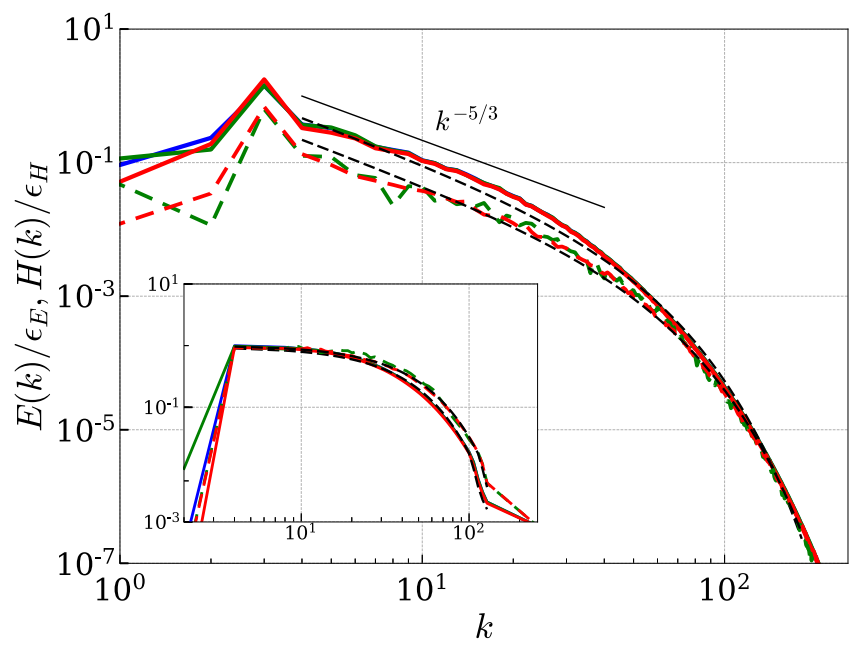

FIG. 3. Normalized energy spectra (solid curves) and helicity spectra (dashed curves) with the same color code as in Fig. 2. In the inset, normalized energy fluxes (solid curves) and helicity fluxes (dashed curves). The black dashed curves correspond to the analytical formulas (40)-(43).

\section{Results of enstrophy fluxes}

The enstrophy fluxes given in Eqs. (17)-(21) have been calculated for $\epsilon_{H}=0,0.1,0.2$. They are found to be insensitive to $\epsilon_{H}$. Therefore only the results corresponding to $\epsilon_{H}=0$ are presented in Fig. 4. The manifestation of this independence is consistent with many numerical results which showed that the forward flux of kinetic energy is also insensitive to kinetic helicity injection at large scale [44]. Crucial changes can be expected when significant imbalance between helical modes of different signs is imposed by helicity forced over a wide range of scales [22].

The flux $\Pi_{\omega>}^{\omega<}$ is positive, suggesting a direct cascade of enstrophy. In the inertial range it obeys a $k^{2}$ scaling law. However, we observe that in the inertial range $\Pi_{\omega>}^{u<}$ is larger than $\Pi_{\omega>}^{\omega<}$, showing that the enstrophy flux due to stretching is larger than the one due to advection. We also observe that

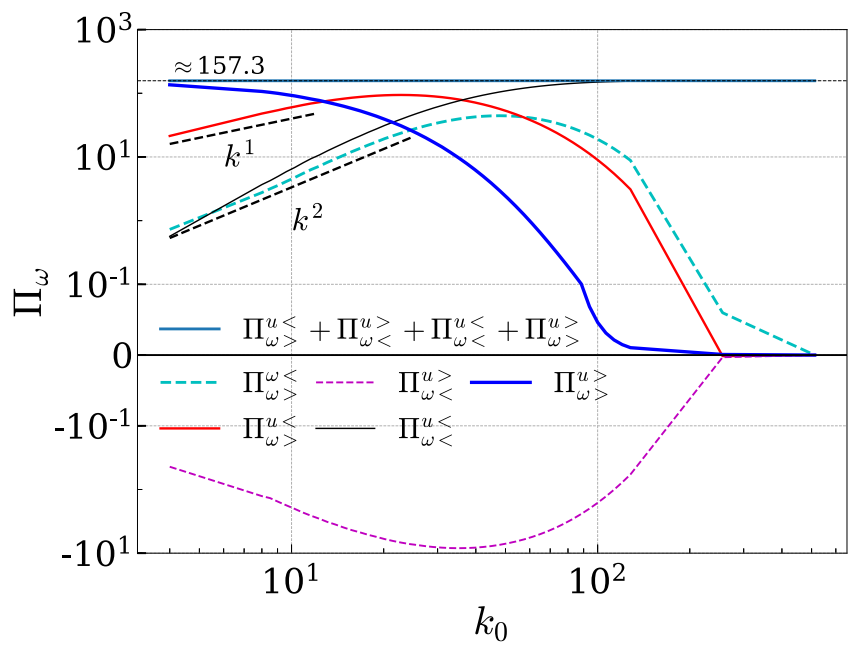

FIG. 4. The five enstrophy fluxes given in Eqs. (17)-(21) and the sum $\Pi_{\omega>}^{u<}+\Pi_{\omega<}^{u>}+\Pi_{\omega<}^{u<}+\Pi_{\omega>}^{u>}$ are plotted for $\epsilon_{E}=0.1$ and $\epsilon_{H}=0$. 
$\Pi_{\omega<}^{u>}$ is always negative, indicating that the small scale velocity fluctuations squeeze (not stretch) the large scale vorticity. It also obeys a $k^{2}$ scaling law in the inertial range.

The flux $\Pi_{\omega<}^{u<}\left(k_{0}\right)$ is an accumulated sum of all the enstrophy transferred from $u^{<}$to $\omega^{<}$up to the radius $k_{0}$. All the wave number shells have positive sign for these transfers, hence $\Pi_{\omega<}^{u<}\left(k_{0}\right)$ is an increasing function of $k_{0}$. Note that $\Pi_{\omega<}^{u<}(\infty)$ is the net enstrophy transfer. The complementary flux, $\Pi_{\omega>}^{u>}\left(k_{0}\right)$, has an opposite behavior; that is, it decreases with $k_{0}$. All the fluxes other than $\Pi_{\omega<}^{u<}\left(k_{0}\right)$ vanish for $k_{0}>k_{d}$ because the fluctuations vanish in this range.

It is found that $\left(\Pi_{\omega>}^{u<}+\Pi_{\omega<}^{u>}+\Pi_{\omega<}^{u<}+\Pi_{\omega>}^{u>}\right)\left(k_{0}\right) \approx 157.3$, independently of $k_{0}$ to a precision of about $10^{-10}$. This value is comparable to the enstrophy dissipation which is about 160.9 (Fig. 2); the difference between the two quantities is $\sum_{k_{0}^{F}<\left|\mathbf{k}^{\prime}\right| \leqslant k_{1}^{F}}\left|k^{\prime}\right|^{2} \operatorname{Re}\left\{\mathbf{u}^{*}\left(\mathbf{k}^{\prime}\right) \cdot \mathbf{F}\left(\mathbf{k}^{\prime}\right)\right\} \approx 3.6$; these computations are consistent with (27).

\section{CONCLUSIONS}

Using direct numerical simulations and varying the injection rate of helicity, we find that injecting helicity does not change the results in terms of energy and helicity spectra and fluxes, and also in terms of enstrophy fluxes. The energy and helicity spectra and fluxes follow rather well the formula of Pao [40] that we extended to helicity. Of course, some correction due to intermittency and bottleneck effect should be added, but these issues are beyond the scope of the present paper. These results are consistent with the predictions that the inertial range properties of hydrodynamic turbulence is independent of kinetic helicity $[13,24]$.

The main objective of this paper is to introduce the derivation of mode-to-mode enstrophy transfers, using which we compute the enstrophy fluxes. There are five different enstrophy fluxes. Four of them, $\Pi_{\omega<}^{u<}, \Pi_{\omega>}^{u<}, \Pi_{\omega<}^{u>}, \Pi_{\omega>}^{u>}$, correspond to vorticity stretching; the fifth one $\Pi_{\omega>}^{\omega<}$ is due to the advection of vorticity by the flow. It is remarkable that in the inertial range $\Pi_{\omega>}^{u<}$ is larger than $\Pi_{\omega>}^{\omega<}$, implying that the enstrophy flux due to vorticity stretching is larger than the one due to advection of vorticity. The sum of the first four fluxes corresponds to the contribution from all velocity modes to all vorticity modes and therefore should not depend on the wave number, which we confirm numerically with a precision better than $10^{-10}$.

\section{ACKNOWLEDGMENTS}

Our numerical simulations have been performed on Shaheen II at the KAUST supercomputing laboratory, Saudi Arabia, under the project k1052. This work was supported by the research grants PLANEX/PHY/2015239 from the Indian Space Research Organisation, India, and by the Department of Science and Technology, India (INT/RUS/RSF/P-03).

[1] A. N. Kolmogorov, The local structure of turbulence in incompressible viscous fluid for very large Reynolds numbers, Dokl. Akad. Nauk SSSR 30, 299 (1941).

[2] A. N. Kolmogorov, Dissipation of energy in locally isotropic turbulence, Dokl. Akad. Nauk SSSR 32, 16 (1941).

[3] A. M. Obukhov, Structure of the temperature field in turbulent flows, Isv. Akad. Nauk. SSSR, Ser. Geogr. Geophys. 13, 58 (1949).

[4] S. Corrsin, On the spectrum of isotropic temperature fluctuations in an isotropic turbulence, J. Appl. Phys. 22, 469 (1951).

[5] M. Lesieur, Turbulence in Fluids (Springer-Verlag, Dordrecht, 2008).

[6] M. K. Verma, Physics of Buoyant Flows: From Instabilities to Turbulence (World Scientific, Singapore, 2018).

[7] R. H. Wasserman, Helical fluid flows, Q. Appl. Math. 17, 443 (1960). 
[8] A. Brissaud, U. Frisch, J. Leorat, M. Lesieur, and A. Mazure, Helicity cascades in fully developed isotropic turbulence, Phys. Fluids 16, 1366 (1973).

[9] J. C. André and M. Lesieur, Influence of helicity on the evolution of isotropic turbulence at high Reynolds number, J. Fluid Mech. 81, 187 (1977).

[10] W. Polifke and L. Shtilman, The dynamics of helical decaying turbulence, Phys. Fluids A 1, 2025 (1989).

[11] F. Waleffe, The nature of triad interactions in homogeneous turbulence, Phys. Fluids A 4, 350 (1992).

[12] N. Yokoi and A. Yoshizawa, Statistical analysis of the effects of helicity in inhomogeneous turbulence, Phys. Fluids A 5, 464 (1993).

[13] Y. Zhou and G. Vahala, Reformulation of recursive-renormalization-group-based subgrid modeling of turbulence, Phys. Rev. E 47, 2503 (1993).

[14] P. D. Ditlevsen and P. Giuliani, Dissipation in helical turbulence, Phys. Fluids 13, 3508 (2001).

[15] Q. Chen, S. Chen, and G. L. Eyink, The joint cascade of energy and helicity in three-dimensional turbulence, Phys. Fluids 15, 361 (2003).

[16] T. Lessinnes, F. Plunian, R. Stepanov, and D. Carati, Dissipation scales of kinetic helicities in turbulence, Phys. Fluids 23, 035108 (2011).

[17] Q. Chen, S. Chen, G. L. Eyink, and D. D. Holm, Intermittency in the Joint Cascade of Energy and Helicity, Phys. Rev. Lett 90, 214503 (2003).

[18] T. Teitelbaum and P. D. Mininni, The decay of turbulence in rotating flows, Phys. Fluids 23, 065105 (2011).

[19] A. Pouquet, J. Baerenzung, P.D. Mininni, D. Rosenberg, and S. Thalabard, Rotating helical turbulence: Three-dimensionalization or self-similarity in the small scales? J. Phys.: Conf. Ser. 318, 042015 (2011).

[20] R. Stepanov, E. Golbraikh, P. Frick, and A. Shestakov, Helical bottleneck effect in 3D homogeneous isotropic turbulence, Fluid Dyn. Res. 50, 011412 (2018).

[21] L. Biferale, S. Musacchio, and F. Toschi, Inverse Energy Cascade in Three-Dimensional Isotropic Turbulence, Phys. Rev. Lett. 108, 164501 (2012).

[22] M. Kessar, F. Plunian, R. Stepanov, and G. Balarac, Non-Kolmogorov cascade of helicity-driven turbulence, Phys. Rev. E 92, 031004(R) (2015).

[23] G. Sahoo and L. Biferale, Energy cascade and intermittency in helically decomposed Navier-Stokes equations, Fluid Dyn. Res. 50, 011420 (2018).

[24] V. Avinash, M. K. Verma, and A. V. Chandra, Field-theoretic calculation of kinetic helicity flux, Pramana J. Phys. 66, 447 (2006).

[25] A. Teimurazov, R. Stepanov, M. K. Verma, S. Barman, A. Kumar, and S. Sadhukhan, Direct numerical simulation of homogeneous isotropic helical turbulence with the TARANG code, Comput. Continuum Mech. 10, 474 (2017) [J. Appl. Mech. Tech. Phys. 59, 1279 (2018)].

[26] M. K. Verma, Statistical theory of magnetohydrodynamic turbulence: Recent results, Phys. Rep. 401, 229 (2004).

[27] A. Alexakis, P. D. Mininni, and A. Pouquet, On the inverse cascade of magnetic helicity, Astrophys. J. 640, 335 (2006).

[28] T. Lessinnes, F. Plunian, and D. Carati, Helical shell models for MHD, Theor. Comput. Fluid Dyn. 23, 439 (2009).

[29] F. Krause and K.-H. Rädler, Mean-Field Magnetohydrodynamics and Dynamo Theory (Pergamon, Oxford, 1980).

[30] H. K. Moffatt, Magnetic Field Generation in Electrically Conducting Fluids (Cambridge University Press, Cambridge, 1978).

[31] D. Sokoloff, P. Akhmetyev, and E. Illarionov, Magnetic helicity and higher helicity invariants as constraints for dynamo action, Fluid Dyn. Res. 50, 011407 (2017).

[32] Y. B. Zeldovich, A. A. Ruzmaikin, and D. D. Sokoloff, Magnetic Fields in Astrophysics (Gordon and Breach, London, 1983).

[33] M. K. Verma, A. G. Chatterjee, R. K. Yadav, S. Paul, M. Chandra, and R. Samtaney, Benchmarking and scaling studies of pseudospectral code Tarang for turbulence simulations, Pramana J. Phys. 81, 617 (2013). 
[34] A. G. Chatterjee, M. K. Verma, A. Kumar, R. Samtaney, B. Hadri, and R. Khurram, Scaling of a fast Fourier transform and a pseudo-spectral fluid solver up to 196608 cores, J. Parallel Distrib. Comput. 113, 77 (2018).

[35] G. Dar, M. K. Verma, and V. Eswaran, Energy transfer in two-dimensional magnetohydrodynamic turbulence: Formalism and numerical results, Physica D 157, 207 (2001).

[36] C. Canuto, M. Y. Hussaini, A. Quarteroni, and T. A. Zang, Spectral Methods in Fluid Dynamics (SpringerVerlag, Berlin, 1988).

[37] J. P. Boyd, Chebyshev and Fourier Spectral Methods, 2nd ed. (Dover, New York, 2003).

[38] D. Carati, O. Debliquy, B. Knaepen, B. Teaca, and M. K. Verma, Energy transfers in forced MHD turbulence, J. Turbul. 7, N51 (2006).

[39] D. Carati, S. Ghosal, and P. Moin, On the representation of backscatter in dynamic localization models, Phys. Fluids 7, 606 (1995).

[40] Y.-H. Pao, Structure of turbulent velocity and scalar fields at large wavenumbers, Phys. Fluids 8, 1063 (1965).

[41] M. K. Verma, A. Kumar, P. Kumar, S. Barman, A. G. Chatterjee, R. Samtaney, and R. A. Stepanov, Energy spectra and fluxes in dissipation range of turbulent and laminar flows, Fluid Dyn. 53, 862 (2018).

[42] G. Falkovich, Bottleneck phenomenon in developed turbulence, Phys. Fluids 6, 1411 (1994).

[43] M. K. Verma and D. A. Donzis, Energy transfer and bottleneck effect in turbulence, J. Phys. A: Math. Theor. 40, 4401 (2007).

[44] A. Alexakis and L. Biferale, Cascades and transitions in turbulent flows, Phys. Rep. 767, 1 (2018). 